\title{
Wie wichtig ist die Orthopädie für die Studenten?
}

Die Universitätsklinika und Medizinischen Hochschulen sind zuständig für die studentische Lehre, Forschung und Patientenversorgung. Die Diskussion um Qualitätsverbesserung in der medizinischen Ausbildung wird seit Jahren geführt (Gulich 1999). Ein Ende ist nicht abzusehen, nachdem die von der vergangenen Bundesregierung beabsichtigte grundlegende Reform des Medizinstudiums vor Ende der vergangenen Legislaturperiode gescheitert ist.

Ziel der ärztlichen Ausbildung ist der wissenschaftliche und praktisch in der Medizin ausgebildete Arzt, der zur eigenverantwortlichen und selbständigen ärztlichen Berufsausübung, zur Weiterbildung, zur ständigen Fortbildung und zur kritischen Bewertung seines Handelns befähigt ist (Juni 1998). Die Ausbildung soll grundlegende Kenntnisse, Fähigkeiten und Fertigkeiten in allen Fächern vermitteln, die für eine umfassende Gesundheitsversorgung der Bevölkerung erforderlich sind. Die Frage, ob die Fertigkeiten im Fach Orthopädie ausreichen, um die umfassende Versorgung der Bevölkerung zu garantieren, wurde wiederholt gestellt (Niethard 1997). Sie stellt sich erneut, da der Bedarf an Kenntnissen ständig steigt, andererseits aber das Angebot für die Vermittlung der orthopädischen Fertigkeiten zur Diskussion gestellt wird. So sind aus einzelnen Universitäten und den zuständigen Ministerien Konzepte zu vernehmen, wonach das Fach Orthopädie nicht mehr überall vertreten sein müsse. Damit würden der Orthopädie mit der Argumentation einer Profilierung medizinischer Ausbildung - in Wahrheit aber unter dem Aspekt des Kostendruckes - die Aufgaben der ärztlichen Ausbildung entzogen.

Die volkswirtschaftliche Bedeutung orthopädischer Erkrankungen ist durch die Statistiken von Kranken- und Rentenversicherungsträgern zu belegen: degenerative Erkrankungen der Stütz- und Bewegungsorgane sind eine Volkskrankheit (Memorandum Orthopädie 1998):

- Unter den Ersterkrankungen mit Arztkontakt stehen die orthopädischen Erkrankungen an erster Stelle.

- Die meisten Arbeitsunfähigkeitstage werden durch Erkrankungen des Skelett- und Muskelsystemes verursacht. Die Krankenkassen registrieren in den letzten 15 Jahren einen deutlichen Anstieg dieser Erkrankungen und der daraus resultierenden Zunahme von Arbeitsunfähigkeit, während Atemwegserkrankungen, Kreislauf- und Verdauungsorgankrankheiten in ihren Auswirkungen stagnieren.

- Nach dem statistischen Jahrbuch überwiegen orthopädische Erkrankungen auch als Ursache der Rentengewährung beim Zugang an Renten wegen Erwerbsund Berufsunfähigkeit.

- Bei den Krankheiten und Behinderungen, die für die Feststellung von Pflegebedürftigkeit nach der neuen Pflegeversicherung anerkannt sind, werden Verluste, Lähmungen oder andere Funktionsstörungen an den Stütz- und Bewegungsorganen zuerst genannt.

- Operative Eingriffe an Knochen und Gelenken stehen in der OP-Statistik nach Operationen an den Augen-

Z. Orthop. 137 (1999) 199-200

(C) 1999 Georg Thieme Verlag Stuttgart · New York linsen an zweiter Stelle (Krankenhausreport 1998, Wissenschaftliches Institut der AOK).

Mit der demographischen Entwicklung wird die volkswirtschaftliche Bedeutung orthopädischer Erkrankungen zunehmen. Folgerichtig wurde die Bone and Joint Decade 2000 bis 2010 ausgerufen (Niethard u. Puhl 1999). Das Ziel dieser Initiative besteht darin, die Relevanz der Erkrankungen und Verletzungen der Haltungs- und Bewegungsorgane für den einzelnen, die Gesellschaft, die Gesundheits- und Sozialsysteme zu verdeutlichen, und gleichzeitig das Bewußtsein zu steigern, daß die Ressourcen für das Management dieser Erkrankungen und Verletzungen effizienter genutzt werden müssen.

Dazu gehört auch eine Intensivierung der studentischen Ausbildung auf dem Gebiet orthopädischer Erkrankungen. Qualitätssicherung im Berufsleben bedeutet Sicherung der Qualität in der Ausbildung. Qualitätssicherung ist Kostenersparnis: der inflationäre Anstieg diagnostischer Leistungen ist zu einem großen Teil auf ungenügende Ausbildung zurückzuführen. Bei der Logik unseres Gesundheitssystemes bedeutet dies in zunehmendem Umfang, daß die Ressourcen durch diagnostischen Aufwand ausgeschöpft sind, bevor therapeutische Maßnahmen greifen können.

Daß das Problem einer ungenügenden orthopädischen Ausbildung von Studenten von großer Bedeutung ist, zeigt eine Studie aus den USA. Freedman u. Bernstein berichten kürzlich im Journal of Bone and Joint Surgery über "The Adequacy of Medical School Education in Musculoskeletal Medicine“. Sie verweisen auf Untersuchungen, wonach $51 \%$ von 255 Allgemeinmedizinern (in den USA gleich family practice physicians) angaben, daß ihre studentische Ausbildung auf dem Gebiet der Orthopädie unzureichend für die Anforderung ihrer Praxis gewesen sei. Weitere Studien zeigten, daß Pädiater die Orthopädie als das Fach bezeichneten, für das sie eine unzureichende Basis erworben hätten. Die Autoren verweisen auf verschiedene Studien, wonach spezielle orthopädische Untersuchungstechniken von Allgemeinärzten nicht beherrscht wurden. So wurden in einer Studie z. B. nur 6\% der chronischen Knieschäden bei Kreuzbandverletzungen von den „Primary Care Physicians“ erkannt. In einer weiteren Studie wurden nur 10\% der nicht-orthopädisch untergebrachten stationären Patienten auch entsprechend untersucht, obwohl $40 \%$ dieser Patienten über Probleme der Haltungs- und Bewegungsorgane klagten.

In ihrer eigenen Studie habe Freedman u. Bernstein Berufsanfängern im ersten Jahr ihrer Tätigkeit Fragen aus dem orthopädischen Fachgebiet vorgelegt, die von einem renommierten Fachkollegium nach den Kriterien der Kompetenz für das orthopädische Gebiet gesichtet worden waren. Danach zeigte es sich, daß 82\% der „Postgraduates" die Kompetenz für fundamentales orthopädisches Wissen vermissen ließen. Die Autoren schließen daraus, daß die studentische Ausbildung auf dem Gebiet der Orthopädie unzureichend ist. Die Befragten der Studie hatten während ihres Studiums insgesamt 2,1 Wochen orthopädische Ausbildung erhalten. Dies dürfte in etwa den 
Verhältnissen in der BRD entsprechen. 33\% der Befragten hatten in der praktischen Arbeit niemals Kontakt mit orthopädischen Problemen gehabt. Diese zeigten bei der Befragung auch die schlechtesten Ergebnisse. Dies dürfte sich für das Wahlfach Orthopädie in der BRD eher noch ungünstiger darstellen.

Bei der hohen Prävalenz von Erkrankungen und Verletzungen der Haltungs- und Bewegungsorgane, die einer alltäglichen Betreuung in der Praxis bedürfen, steht die $\mathrm{Be}$ deutung einer umfassenden Kompetenz des angehenden Arztes für orthopädische Erkrankungen außer Zweifel. Eine Einschränkung des orthopädischen Lehrstoffes an Universitäten und Medizinischen Hochschulen verbietet sich demnach von selbst. Es ist vielmehr zu fordern, daß die mit der Ausbildung befaßten Institutionen ihre Curricula überprüfen und die theoretische und praktische Ausbildung der Studenten im Fach Orthopädie verbessert werden. Neue Konzepte wie das ,problemorientierte Lernen" vermögen hier nur dann Abhilfe zu schaffen, wenn das Basiswissen für orthopädische Erkrankungen ausreichend vermittelt wird. Es ist zu befürchten, daß sich die derzeit ungenügende studentische Ausbildung im Gebiet Orthopädie besonders nachteilig auswirken wird, wenn durch Änderungen im Gesundheitssystem (PrimärarztSystem) orthopädische Erkrankungen in Fachbereiche verwiesen werden, wo diese Kompetenz bisher nicht vorhanden ist. Spätestens dann ist es erforderlich, das Gesamtkonzept der medizinischen Ausbildung zu überdenken und an den Bedürfnissen der alltäglichen Praxis und des „Gesundheitsmarktes“ zu orientieren.

F. U. Niethard, Aachen

\section{Literatur}

Freedman, K. B., J. Bernstein: The Adequacy of Medical School Education in Musculoskeletal Medicine. J. Bone Jt. Surg. 80-A (1998) 1421-1427

Gulich, M. S.: Medizinische Ausbildung: Irrtümer - und kein Ende? Deutsches Ärzteblatt 96 (1999) B-805-808

Krankenhausreport 1998: Wissenschaftliches Institut der AOL (WiDO)

Jani, L.: Neue Approbationsordnung und Orthopädie - Editorial. Z. Orthop. 136 (1998) 1-2

Memorandum Orthopädie des Präsidiums der Deutschen Gesellschaft für Orthopädie und Traumatologie. Stork, Bruchsal 1998

Niethard, F. U.: Qualitätssicherung - Editorial. Z. Orthop.. 135 (1997) 93-94

Niethard, F. U., W. Puhl: The Bone and Joint Decade 2000-2010-Editorial. Z. Orthop. 137(1999) 1 